\title{
Oscillations to hidden photon in reactor and accelerator ex- periments
}

\author{
Dmitry Gorbunov ${ }^{1, *}$ \\ ${ }^{1}$ Institute for Nuclear Research of the Russian Academy of Sciences, \\ 60th October Anniversary prospect, 7a, Moscow 117312, Russia
}

\begin{abstract}
Possible connection between visible and hidden Worlds of particle physics is provided by the vector portal interaction, that is kinetic mixing between ordinary (visible) and hidden photons. If sufficiently light, the hidden photon can be produced (and absorbed) by ordinary matter in a Compton-like processes, which naively are insensitive to the hidden photon mass. In this talk we study the evolution of the two vector boson system, which exhibits oscillations similar to the well-known neutrino oscillations. We show that, provided by the environment matter, this quantum phenomenon significantly suppresses the hidden photon production in particle collisions. The ordinary photon is effectively massive in the media. The suppression is important for hidden photon lighter than photon in the corresponding media (detector, beam dump material etc) and grows with decrease of the hidden photon mass making all the accelerator and reactor experiments absolutely insensitive to the models with light photons. The dedicated study is required for the case of the degenerate photon and hidden photon, when, on the contrary, the hidden photon production can be amplified with respect to the Compton process.
\end{abstract}

\section{Introduction}

Two statements about particle physics phenomenology are widely accepted at present. First, the Standard Model of particle physics (SM) nicely explains almost all results of particle physics experiments. Second, we definitely need new particle physics. The reason for the first statement is the PDG gross-book [1] with numerous references on the experiments, all consistent with theoretical predictions obtained within the SM. The reason for the second statement is that we have several phenomena beyond the SM: neutrino oscillations (the only direct evidence of new physics), baryon asymmetry of the Universe, dark matter, flatness and homogeneity of the present Universe. On the theoretical side there is one more very relevant statement: any new heavy particles contribute quantum corrections to the SM Higgs boson mass, $m_{h}$, lifting it up to the energy scale of new physics. However, miraculously in this sense, we found its mass at the electroweak scale, $m_{h} \sim E_{E W}$.

Among the guesswork suggested so far one can recognize the logically possible and rather attractive option: all new particles responsible for the aforementioned problems are actually light, at or even below the electroweak scale $E_{E W}$, and consequently the SM Higgs boson mass is safe. The natural question of 'why so far have we no evidences for such light new

*e-mail: gorby@ms2.inr.ac.ru 
particles ?' can be successfully answered if the new particles only feebly couple to the SM ones. Hence, they are SM gauge singlets and not low-energy remnants of the Grand Unified Theories. Even in the SM we have examples of a feeble interaction provided by the Yukawa couplings of the first generation of SM fermions.

For the new SM singlets possibly the most promising variant is that associated with the so-called portal-like couplings, which are distinguished being the only renormalizable interactions including the SM fields and new fields singlets with respect to the SM gauge group. In what follows we concentrate in a particular portal with new particles of (sub) GeV masses, so a disclaimer is in order. Namely, there are no general theoretical motivation for the new particles to be exactly at this scale. However, and it is general, for all the feebly coupled particles the best place to show up is the intensity frontier experiments. Moreover, there are many concrete beyond the SM theories which suggest such theoretical motivations. The present talk is partly based on some finished papers [2, 3] and work in progress [4], and aimed at stimulating collaborations of the relevant intensity frontier experiments (TEXONO, NEOS, DANSS, NA64, SHiP, DUNE, T2K, MicroBooNE, etc) to perform searches for the light hidden photons following the theoretical analysis presented below, which accounts for oscillations between photon and hidden photon very similar to neutrino oscillation phenomena.

\section{Vector portal to the hidden World}

There are three portals: dimension-4 operators describing interactions between the SM fields and new SM singlets. The scalar portal

$$
\mathcal{L}_{\text {scalar portal }}=-\beta H^{\dagger} H S^{\dagger} S
$$

couples the SM singlet $S$ to the SM Higgs weak doublet $H$. This model provides with the simplest yet viable dark matter candidate. The spinor portal

$$
\mathcal{L}_{\text {spinor portal }}=-y \bar{L} \tilde{H} N
$$

describes interaction between the SM lepton doublet $L$, the Higgs conjugate field $\tilde{H}=\epsilon H^{*}$ and the hidden fermion $N$. The new fermions play role of the right-handed neutrino components, and if they are massive (Majorana type mass), the spinor portal becomes the main ingredient of the seesaw type I mechanism generating SM neutrino masses. Finally, the vector portal

$$
\mathcal{L}_{\text {vector portal }}=-\frac{\epsilon}{2} B_{\mu \nu}^{U(1)_{Y}} B_{\mu \nu}^{U(1)^{\prime}}
$$

yields kinetic mixing between the SM Abelian $U(1)_{Y}$ gauge field and the gauge field of the hidden Abelian group $U(1)^{\prime}$. The low-energy effective couplings with photon and Z-boson depend on the hidden gauge sector and details of the Higgs mechanism operating in the hidden sector and making the hidden photon massive.

In this talk we consider only kinetic mixing between hidden photon and visible photon. This mixing can be nothing less but a bridge to the dark matter World. The phenomenologically and cosmologically acceptable example is given by the following lagrangian [5],

$$
\mathcal{L}_{\mathrm{DM}+\text { mediator }}=\bar{\Psi}\left(i \gamma^{\mu} \partial_{\mu}-e^{\prime} \gamma^{\mu} A_{\mu}^{\prime}-m_{\Psi}\right) \Psi-\frac{1}{4} A_{\mu \nu}^{\prime} A^{\prime \mu \nu}+\frac{m_{A^{\prime}}^{2}}{2} A_{\mu}^{\prime} A^{\prime \mu}+\epsilon A_{\mu}^{\prime} \partial_{v} B^{\mu \nu},
$$

where at $m_{\Psi}>m_{A^{\prime}} \sim 1 \mathrm{GeV}$ the fermion $\Psi$ can serve as the thermal dark matter, similar to the standard WIMPs. The hidden photons mix with ordinary photons and hence are unstable. In the early Universe they must decay before the Big Bang Nucleosynthesis, $\tau_{A^{\prime}}<1 \mathrm{~s}$, which 
imposes the following constraint on the model parameters, $\epsilon^{2}\left(m_{A^{\prime}} / 1 \mathrm{GeV}\right) \gtrsim 10^{-21}$. With some extensions the model may explain the muon $(g-2)$ discrepancy and anomalies in indirect searches for the Galactic dark matter by Pamela, Fermi, etc.

The hidden photon phenomenology is determined by mixing with visible photons. One expects that both production and decay of the hidden photon proceed through the virtual photon, the both rates scale as $\propto \epsilon^{2}$. If kinematically allowed, the hidden photon decays into charge lepton pair $l^{+} l^{-}$with rate

$$
\Gamma_{A^{\prime}}^{l^{+} l^{-}}=\frac{1}{3} \alpha_{\mathrm{QED}} m_{A^{\prime}} \epsilon^{2} \sqrt{1-\frac{4 m_{l}^{2}}{m_{A^{\prime}}^{2}}}\left(1+\frac{2 m_{l}^{2}}{m_{A^{\prime}}^{2}}\right) .
$$

The decay rate into hadrons can be accurately described by making use of the well-measured ratio

$$
R(\sqrt{s})=\frac{\sigma\left(e^{+} e^{-} \rightarrow \text { hadrons }\right)}{\sigma\left(e^{+} e^{-} \rightarrow \mu^{+} \mu^{-}\right)}
$$

so that

$$
\Gamma_{A^{\prime}}^{\text {hadrons }}=\frac{1}{3} \alpha_{\mathrm{QED}} m_{A^{\prime}} \epsilon^{2} \cdot R\left(m_{A^{\prime}}\right) .
$$

The hadronic branchings and the hidden photon decay length $c \tau_{A^{\prime}}$ are presented in Fig. 1, see
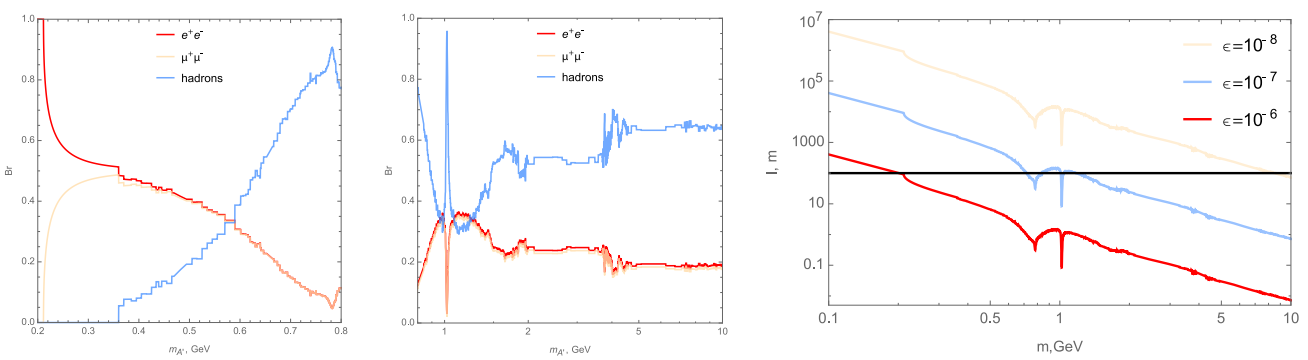

Figure 1. Hadronic branching ratios (left and middle panels) and hidden photon decay length (right panel) for the hidden photon at GeV-mass scale [2].

Ref. [2] for details. As to the hidden photon production, it can emerge in decays of unstable hadrons, wherever photons may show up in the final state: e.g., in 2-photon decays of neutral mesons, $\pi^{0}, \eta^{0}$, or in 2-body decays of vector mesons $\rho^{ \pm}, \rho^{0}, \omega$ into pions. The branching ratio of neutral pion reads,

$$
\mathrm{Br}_{\pi^{0} \rightarrow A^{\prime} \gamma} \simeq 2 \epsilon^{2}\left(1-\frac{m_{A^{\prime}}^{2}}{m_{\pi^{0}}^{2}}\right)^{3} \operatorname{Br}_{\pi^{0} \rightarrow \gamma \gamma},
$$

and similar expressions may be written for the branchings of vector mesons.

In the accelerator experiments the hidden photon can be produced by the energetic particles scattering off target. For the (sub)GeV hidden photons the complementary channels are electron and proton bremsstrahlung for the electron and proton beam, respectively. Searches for the hidden photons have been performed with high-intensity accelerators of JPARC, CERN SPS, Protvino, Fermilab and are included in the physical programs of relevant future projects like SHiP, DUNE, upgrade T2K, etc. 


\section{Hidden photons in reactor experiments}

Sufficiently light hidden photons can be produced at nuclear power plants, where numerous photons of $\mathrm{MeV}$ energy range scatter off the reactor core material. In this Compton-like processes conversion $\gamma \rightarrow X$ (absorption of the incident photon and subsequent emission of the hidden photon) may happen; hereafter we use both $A^{\prime}$ and $X$ to denote the hidden photon. Then the hidden photon escapes the reactor and may be observed in the external detector where the inverse process $X \rightarrow \gamma$ may happen. The signature is called 'light shining through the wall' in the framework of experiments aimed at axion hunting. Actually, the $\mathrm{MeV}$-photon kicks out an electron, the process mimics electron antineutrino scattering $\bar{v}+$ $e^{-} \rightarrow e^{-}$. The latter processes is exploited in many experiments dedicated to measurements of reactor antineutrino flux. Hence, all such experiments, e.g. TEXONO, NEOS, DANSS, are actually sensitive to the models with hidden photons we are interested in.

At a nuclear power plant of thermal power $T h P$ the total differential photon flux per second is about

$$
\frac{d N_{\gamma}}{d\left(E_{\gamma} / 1 \mathrm{MeV}\right)} \approx 0.6 \times 10^{21} \times \frac{T h P}{\mathrm{GW}} \times \exp ^{-\frac{E_{\gamma}}{0.91 \mathrm{MeV}}} .
$$

Constraints on the model parameters from analysis of the results of TEXONO and NEOS neutrino reactor experiments obtained in Ref. [6] are presented in Fig. 2 One observes the

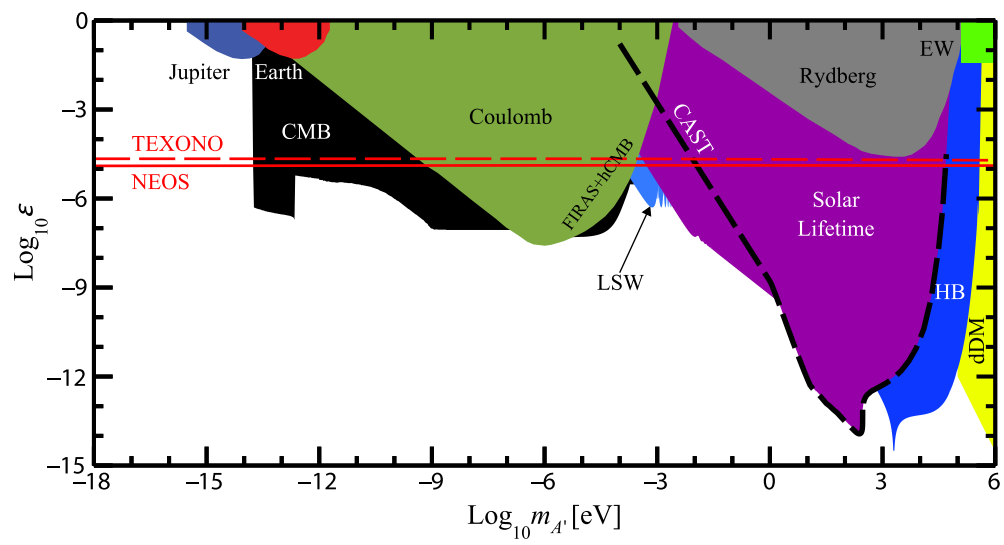

Figure 2. Limits on the mixing and hidden photon mass presented in Ref. [6].

expected tendency: for light hidden photon the upper limit on the mixing $\varepsilon$ does not depend on hidden photon mass $m_{A^{\prime}}$. However, at the low-mass end of the range, where the presented limits surpass those from astrophysics and cosmology, the numbers are too exotic. Namely, for the particle of $10^{-18} \mathrm{eV}$ the corresponding Compton wave-length is about $\lambda=2 \pi /\left(10^{-18} \mathrm{eV}\right)=10^{14} \mathrm{~cm}$, which is about 10 astronomical units! It is weird: the reactor size is of order meters. This region of parameters space seems irrelevant for the reactor experiments, the Compton processes are too fast and the reactor core is too small to produce the quantum wave-packet of the Solar system size. There is something wrong with theoretical description.

In the limit of ultralight hidden photon, $m_{A^{\prime}} \lll E$, we have a system with two extremely light (photon and hidden photon) particles which mix with each other. Similar situation we have in neutrino physics: we know neutrinos are massive yet ultrarelativistic in all the situations where we can observe them. We successfully describe the evolution of neutrino system by making use of the oscillation approximation. And it must be applied to the case at hand as well. 


\section{Oscillations between visible and hidden photons}

Let us start with general lagrangian describing massive hidden photon $X_{\mu}$, its kinetic mixing with ordinary photon $A_{\mu}$ and latter interaction with electromagnetic current $j_{e m}^{\mu}$,

$$
\mathcal{L}=-\frac{1}{4} F_{\mu \nu}^{2}-\frac{1}{4} X_{\mu \nu}^{2}-\frac{\epsilon}{2} X_{\mu \nu} F^{\mu v}+\frac{m_{X}^{2}}{2} X_{\mu}^{2}-e A_{\mu} j_{e m}^{\mu}
$$

To apply the oscillation approach it is convenient to change the variable $X_{\mu} \rightarrow X_{\mu}+\epsilon A_{\mu}$. It makes the kinetic term diagonal, transferring the mixing to the mass terms, but keeping $X_{\mu}$ sterile with respect to the SM electromagnetic interaction,

$$
\mathcal{L}=-\frac{1}{4} F_{\mu \nu}^{2}-\frac{1}{4} X_{\mu \nu}^{2}+\frac{m_{X}^{2}}{2}\left(X_{\mu}+\epsilon A_{\mu}\right)^{2}-e A_{\mu} j_{e m}^{\mu}+O\left(\epsilon^{2}\right)
$$

At small mixing $\epsilon \ll 1$ the system (1) reminds that of neutrinos, and the system evolution is very similar. It is illustrated in Fig. 3. The external current produces the photon state (green
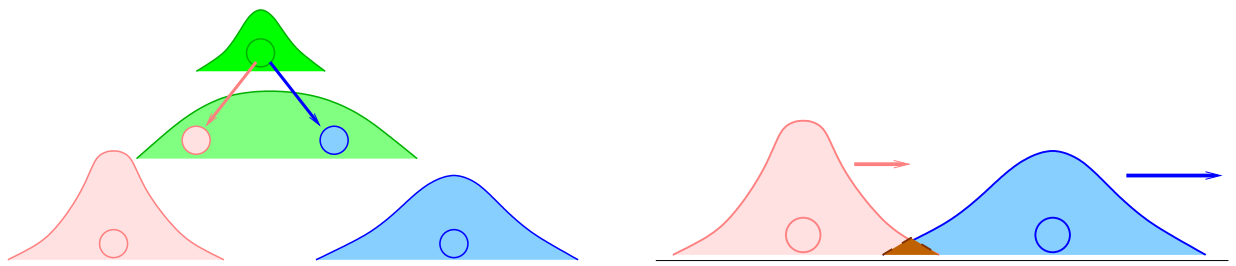

Figure 3. Evolution of the quantum wave-packet of the photon system in vacuum.

color) which is not the eigenstate of the system (1). The eigenstates (pink and blue colors) are linear combinations of photon and hidden photon. They propagate with different velocities, which induces oscillations among the gauge eigenstates (photon and hidden photon). In case of propagation in vacuum, the probability to observe the hidden photon at distance $L$ from the photon source reads

$$
P(A \rightarrow X)=4 \epsilon^{2} \sin ^{2}\left(\frac{m_{X}^{2} L}{4 E}\right) .
$$

Inside the nuclear reactor the photons are produced by decaying fission fragments with typical lifetime $\tau=10^{-12}-10^{-11} \mathrm{~s}$. Hence, the initial width of the quantum wave-packet $\sigma \sim 1 / \tau \sim$ $0.03-0.3 \mathrm{~cm}$ is much shorter than the oscillation length

$$
L_{o s c} \approx 2.5 \mathrm{~cm} \times \frac{E_{\gamma}}{1 \mathrm{MeV}} \frac{(10 \mathrm{eV})^{2}}{m_{X}^{2}},
$$

that justifies the use of the oscillation approximation (in matter the width of the initial packet is even shorter). At the same time, the mass eigenstates become separated due to different velocities, which spoils the coherence at the distance

$$
l_{c o h} \sim 6 \times 10^{8-9} \mathrm{~cm} \times\left(\frac{E_{\gamma}}{1 \mathrm{MeV}}\right)^{2} \frac{(10 \mathrm{eV})^{2}}{m_{X}^{2}} \gg L_{o s c}
$$

where oscillations terminate and the two mass-eigenstates propagate farther without any interference. In matter $L_{c o h}$ is shorter because of photon interactions but still (3) is valid for the case of reactor photons. 
In case of neutrino oscillations the matter effect becomes important in many situations, and similar conclusion is valid for the photon oscillations as well. The oscillations have been studied in case of solar photons [7]. First, photon rescatters in matter, which delays its propagation. This can be effectively described by introducing non-zero mass. Typically, the reactor core is filled with water, hence the photon of $E_{\gamma} \sim 1-10 \mathrm{MeV}$ gains mass of about

$$
m_{\gamma} \sim 20 \mathrm{eV}
$$

Then in matter the eigenvalues change, replacing

$$
m_{X}^{2} \rightarrow \Delta m^{2} \equiv \sqrt{\left(m_{X}^{2}-m_{\gamma}^{2}\right)^{2}+4 \epsilon^{2} m_{X}^{4}}
$$

in eq. (2). Second, photon can be absorbed in matter, and in water the absorption length for $E_{\gamma} \sim 1-10 \mathrm{MeV}$ is about

$$
1 / \Gamma \simeq 10 \mathrm{~cm},
$$

that is smaller than the reactor size. At the distance exceeding absorption length, $L \gg 1 / \Gamma$, the probability to observe the hidden photon approaches $[3,7]$

$$
P=\epsilon^{2} \times \frac{m_{X}^{4}}{\left(\Delta m^{2}\right)^{2}+E_{\gamma}^{2} \Gamma^{2}} .
$$

In the nuclear reactor there is a low absorption regime,

$$
E_{\gamma} \Gamma \approx 2 \times\left(\frac{E_{\gamma}}{1 \mathrm{MeV}}\right)\left(\frac{10 \mathrm{~cm}}{1 / \Gamma}\right) \mathrm{eV}^{2} \ll m_{\gamma}^{2} \sim(20 \mathrm{eV})^{2},
$$

which yields: $P=\epsilon^{2}$, the Compton result for heavy hidden photons, $m_{X} \gg m_{\gamma} ; P=$ $\epsilon^{2} \times\left(m_{X} / m_{\gamma}\right)^{4}$, a strong suppression of production of light hidden photons, $m_{X} \ll m_{\gamma}$; possible amplification, upto $P=10^{5} \times \epsilon^{2}$ for the degenerate case, $m_{X}=m_{\gamma}$, that is resonance. The hidden photon flies away form the reactor and can oscillate back into photon inside the detector of size $L \gg 1 / \Gamma$ with probability (4) where $\Gamma \equiv 0$. Hence, the expected number of 'shining through the wall' photons scales as $N_{\text {sig }} \propto \epsilon^{2} \times \epsilon^{2}$.

Within the oscillation approach one can analyze the results of neutrino reactor experiments and place the limit on model parameters given the full consistency of these results with neutrino oscillations. The investigation [3] revealed that mostly sensitive experiment is TEXONO [8], which allows one to constrain the model parameter space as depicted in Fig. 4. One observes the decrease of sensitivity to light hidden photons because of strong suppression of oscillations in this case. The reason is in intensive rescattering of photon in matter which collapses the quantum wave function and prevents development of the hidden photon state.

The case of resonance, $m_{\gamma}=m_{X}$, requires a special study, with proper account of the material distribution inside both source (reactor core) and detector. The point is that formulas above are valid for the homogeneous case only, but can be easily modified for the general case, e.g. of composite source or multi-layer detector [4]. It requires a good knowledge of the geometry and inner structure of both sites and hence this study must be performed by the corresponding collaborations.

\section{Accelerator experiments}

Light hidden photons, $m_{X}<1 \mathrm{MeV}$, can be searched for with beam-target facilities, NA64 at CERN [9] provides an example of the dedicated experiment. Here electrons of $E_{e}=100 \mathrm{GeV}$ 


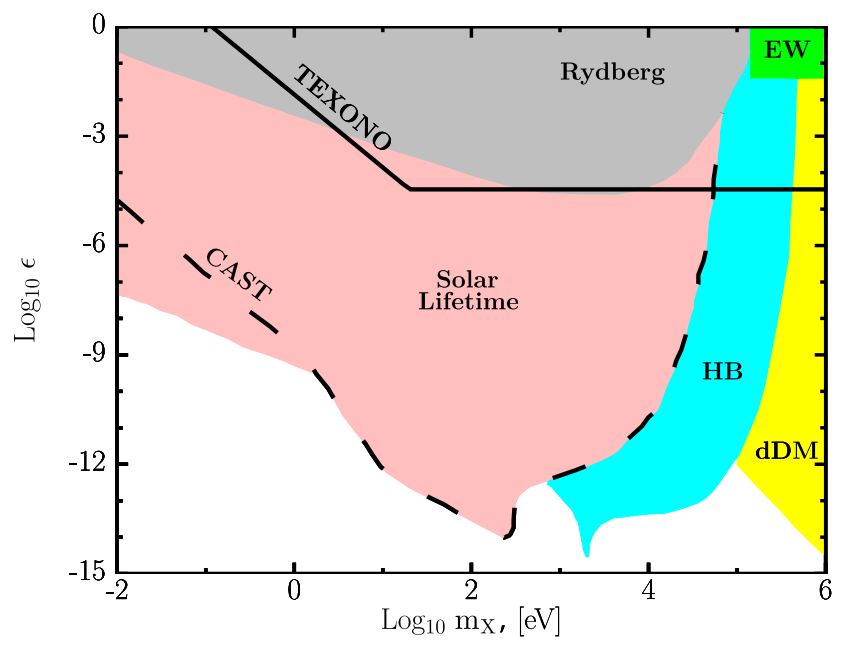

Figure 4. Limits on the mixing from analysis of the TEXONO data, see Ref. [3] for details.

heat the lead dump and the downstream electromagnetic calorimeter collects the energy. For each incident electron an energy loss exceeding $50 \mathrm{GeV}$ would indicate a production of some hidden particles carrying away the corresponding energy.

Ultralight hidden photons can be produced in NA64 experiment by oscillations of the secondary photons inside the lead dump. Here the oscillation length is about

$$
L_{o s c} \approx 25 \mathrm{~cm} \times \frac{E_{\gamma}}{100 \mathrm{GeV}} \frac{(1 \mathrm{keV})^{2}}{m_{X}^{2}}
$$

and photon mass and absorption length are

$$
m_{\gamma} \simeq 60 \mathrm{eV}, \quad 1 / \Gamma=1 \mathrm{~cm} .
$$

Hence, in opposite to the reactor case considered above, the photon system is in the highabsorption regime,

$$
E_{\gamma} \Gamma \simeq\left(\frac{E_{\gamma}}{100 \mathrm{GeV}}\right)\left(\frac{1 \mathrm{~cm}}{1 / \Gamma}\right)(1 \mathrm{keV})^{2} \gg m_{\gamma}^{2} \sim(60 \mathrm{eV})^{2} .
$$

Therefore, for $m_{X}>1 \mathrm{keV}$ we recover the Compton result, and for $m_{X}<1 \mathrm{keV}$ the production process gets strongly suppressed, $P=\epsilon^{2} \times\left(m_{X} / 1 \mathrm{keV}\right)^{4}$. This behavior is similar to that in case of neutrino reactor experiments. However, there is no chance to get advantage of the resonance $m_{X}=m_{\gamma}$ in the system: at this point the absorption term in denominator of (4) dominates over the mass term, see (5), which yields a suppression rather than amplification.

One concludes, that NA64 looses sensitivity to the model starting from $m_{X}=1 \mathrm{keV}$. Note, that one can not make the resonance relevant in this setup by changing the absorber (dump). Indeed, both $\Gamma$ and $m_{\gamma}^{2}$ scales linear with the material density, and so the hierarchy between the two terms in denominator of (4) remains intact. It can be changed for the photons of lower energy, $E \sim 1 \mathrm{GeV}$ with the same the lead dump. Unfortunately, this option seems unrealistic for the present configuration of NA64.

However, low-energy photons are available in other experiments. In particular, proton beam-dumps generically provide with $\mathrm{sub}(\mathrm{GeV})$ photons via decays of low-energy neutral 
pions produced by incident protons on target. The option can be realized within the SHiP project at CERN and DUNE near detector at Fermilab. In particular, at SHiP with incident protons of $E=400 \mathrm{GeV}$ and molybdenum-tungsten dump [10], one estimates for typical $E_{\gamma} \simeq 10 \mathrm{GeV}$

$$
m_{\gamma} \simeq 100 \mathrm{eV}, \quad \text { and } \quad 1 / \Gamma \simeq 0.5 \mathrm{~cm} .
$$

The oscillation length then is

$$
L_{o s c} \approx 5 \mathrm{~cm} \times \frac{E_{\gamma}}{10 \mathrm{GeV}} \frac{(700 \mathrm{eV})^{2}}{m_{X}^{2}}
$$

and the system is again in high-absorption regime,

$$
E_{\gamma} \Gamma \approx\left(\frac{E_{\gamma}}{10 \mathrm{GeV}}\right)\left(\frac{0.5 \mathrm{~cm}}{1 / \Gamma}\right)(700 \mathrm{eV})^{2} \gg m_{\gamma}^{2} \sim(100 \mathrm{eV})^{2} .
$$

The SHiP sensitivity to the model parameters rapidly drops width decreasing hidden photon mass starting from $m_{X} \simeq 700 \mathrm{eV}$. The resonance becomes relevant for the photons of $E_{\gamma} \lesssim$ $250 \mathrm{MeV}$ coming from decays of low-energy neutral pions $E_{\pi} \lesssim 0.5 \mathrm{GeV}$, which are produced by the hadronic shower developing in the dump. With this source of hidden photons one expects the amplification of hidden photon production at the resonance $m_{X}=m_{\gamma} \approx 100 \mathrm{eV}$.

\section{Conclusion}

To summarize, we show that production and detection of ultralight hidden photons must be described via oscillations, similar to the neutrino oscillations. For reactor and accelerator experiments this generically results in decrease of sensitivity with respect to the estimate based on the Compton process. The hidden photon production probability changes as

$$
P=\epsilon^{2} \longrightarrow P=\epsilon^{2} \times\left(\frac{m_{X}}{m_{\text {crit }}}\right)^{4}, \text { where } m_{\text {crit }}^{2}=\max \left[m_{\gamma}^{2}, E_{\gamma} \Gamma\right],
$$

so the the sensitivity to light vectors is lost. The production can be amplified in case of

$$
m_{X}^{2}=m_{\gamma}^{2} \gtrsim E_{\gamma} \Gamma
$$

which requires a special study, better to be performed by the experimental collaborations.

The work was supported by the RSF grant 17-12-01547.

\section{References}

[1] C. Patrignani et al. (Particle Data Group), Chin. Phys. C40, 100001 (2016)

[2] D. Gorbunov, A. Makarov, I. Timiryasov, Phys. Rev. D91, 035027 (2015), 1411.4007

[3] M. Danilov, S. Demidov, D. Gorbunov (2018), 1804 . 10777

[4] S. Demidov, S. Gninenko, D. Gorbunov (2018), 18XY . ZZZZZ

[5] M. Pospelov, A. Ritz, M.B. Voloshin, Phys. Lett. B662, 53 (2008), 0711.4866

[6] H. Park, Phys. Rev. Lett. 119, 081801 (2017), 1705.02470

[7] J. Redondo, JCAP 1507, 024 (2015), 1501. 07292

[8] M. Deniz et al. (TEXONO), Phys. Rev. D81, 072001 (2010), 0911.1597

[9] D. Banerjee et al. (NA64), Phys. Rev. D97, 072002 (2018), 1710.00971

[10] M. Anelli et al. (SHiP) (2015), 1504.04956 